\title{
The abundance of $\mathrm{HNCO}$ and its use as a diagnostic of environment
}

\author{
D. M. Tideswell ${ }^{1}$, G. A. Fuller ${ }^{1}$, T. J. Millar², and A. J. Markwick ${ }^{1}$ \\ 1 Jodrell Bank Centre for Astrophysics, Alan Turing Building, School of Physics and Astronomy, The University of Manchester, \\ Oxford Road, Manchester M13 9PL, UK \\ e-mail: G.Fuller@manchester.ac.uk \\ 2 Astrophysics Research Centre, School of Mathematics and Physics, Queen's University Belfast, Belfast, BT7 1NN, UK
}

Received 18 August 2008 / Accepted 22 September 2009

\section{ABSTRACT}

\begin{abstract}
Aims. We aim to investigate the chemistry and gas phase abundance of $\mathrm{HNCO}$ and the variation of the $\mathrm{HNCO} / \mathrm{CS}$ abundance ratio as a diagnostic of the physics and chemistry in regions of massive star formation.

Methods. A numerical-chemical model has been developed which self-consistently follows the chemical evolution of a hot core. The model comprises of two distinct stages. The first stage follows the isothermal, modified free-fall collapse of a molecular dark cloud. This is immediately followed by an increase in temperature which represents the switch on of a central massive star and the subsequent evolution of the chemistry in a hot, dense gas cloud (the hot core). During the collapse phase, gas species are allowed to accrete on to grain surfaces where they can participate in further reactions. During the hot core phase surface species thermally desorb back in to the ambient gas and further chemical evolution takes place. For comparison, the chemical network was also used to model a simple dark cloud and photodissociation regions.

Results. Our investigation reveals that HNCO is inefficiently formed when only gas-phase formation pathways are considered in the chemical network with reaction rates consistent with existing laboratory data. This is particularly true at low temperatures but also in regions with temperatures up to $\sim 200 \mathrm{~K}$. Using currently measured gas phase reaction rates, obtaining the observed $\mathrm{HNCO}$ abundances requires its formation on grain surfaces - similar to other "hot core" species such as $\mathrm{CH}_{3} \mathrm{OH}$. However our model shows that the gas phase HNCO in hot cores is not a simple direct product of the evaporation of grain mantles. We also show that the $\mathrm{HNCO} / \mathrm{CS}$ abundance ratio varies as a function of time in hot cores and can match the range of values observed. This ratio is not unambiguously related to the ambient UV field as been suggested - our results are inconsistent with the hypothesis of Martín et al. (2008, ApJ, 678, 245). In addition, our results show that this ratio is extremely sensitive to the initial sulphur abundance. We find that the ratio grows monotonically with time with an absolute value which scales approximately linearly with the $\mathrm{S}$ abundance at early times.
\end{abstract}

Key words. astrochemistry - stars: formation

\section{Introduction}

The molecule HNCO (isocyanic acid) was first detected in the interstellar medium over 30 years ago (Snyder \& Buhl 1972) in the Sgr B2 molecular cloud complex where its distribution was found to be spatially extended with relatively strong emission. Since its discovery, $\mathrm{HNCO}$ has been subsequently observed in several other molecular clouds, including the dark cloud TMC-1 (e.g. Brown 1981; Goldsmith et al. 1982; Jackson et al. 1984). In the survey of 18 molecular clouds conducted by Jackson et al. (1984), HNCO emission was detected in seven sources with an average excitation temperature of $12 \mathrm{~K}$, suggesting that this molecule was tracing cold gas. Jackson et al. (1984) proposed that $\mathrm{HNCO}$ was a dense gas tracer due to the coincidence of the emission with regions of high density $\left(n \gtrsim 10^{6} \mathrm{~cm}^{-3}\right)$. A larger survey of 81 molecular clouds was conducted by Zinchenko et al. (2000). They reported a $70 \%$ detection rate with typical fractional abundances relative to the total hydrogen number density, $n_{\mathrm{H}}$, of $\sim 10^{-9}$ and a wide range of rotational temperatures from $10 \mathrm{~K}$ to $500 \mathrm{~K}$. Three of the objects were mapped and showed the emission region to be compact and centrally peaked.

Isocyanic acid has also been detected in hot cores (HCs), the sites of early stage, high mass star-formation including
G34.3+0.15 (MacDonald et al. 1996), W3( $\left.\mathrm{H}_{2} \mathrm{O}\right)$ (Helmich \& van Dishoeck 1997) and Sgr B2; in the N, M and NW cores (Nummelin et al. 2000). A survey towards seven high-mass star forming regions by Bisschop et al. (2007) identified HNCO with fractional abundances relative to $n_{\mathrm{H}}$ of between $6.4 \times 10^{-9}$ and $5.4 \times 10^{-8}$ and excitation temperatures from $64 \mathrm{~K}$ to $278 \mathrm{~K}$. The observations suggested that $\mathrm{HNCO}$ can also trace much warmer gas - consistent with the findings of Zinchenko et al. (2000) and as such could be a potential indicator of star formation activity.

A recent multitransition study of 13 molecular clouds towards the Galactic Centre was conducted by Martín et al. (2008). The sources were selected to represent a range of physical conditions and included photodissociation regions (PDRs) along with HCs and giant molecular clouds. Martín et al. (2008) compared the CS abundance, a species used as a dense gas tracer and regarded as a tracer of star formation sites (Bronfman et al. 1996), to that of HNCO for each of the sources. They found that the ratio of the abundance of $\mathrm{HNCO}$ to that of CS (as traced by ${ }^{13} \mathrm{CS}$ ) was smaller for regions in which the FUV radiation is enhanced (PDRs), perhaps indicating that HNCO is more sensitive than CS to FUV photodissociation. The $\mathrm{HNCO} / \mathrm{CS}$ ratio was systematically smaller in PDR-like regions by approximately two orders of magnitude than in the giant clouds. HCs 
were found to have intermediate values. It was therefore proposed that the $\mathrm{HNCO} / \mathrm{CS}$ abundance ratio may provide a useful tool in distinguishing between shock and radiation (FUV) activity in molecular clouds. However, the Martín et al. (2008) survey only observed a limited sample towards the Galactic Centre and before the usefulness of this possible tracer can be established more complete surveys and a theoretical understanding of the processes involved are desirable.

Since the detection of HNCO there has been much speculation as to how this molecule forms but there have been few chemical models developed which incorporate an HNCO reaction network. Some authors have considered formation only in the gas phase (e.g. Turner et al. 1999; Iglesias 1977) whilst others have modelled the molecule's formation on grain surfaces (e.g. Garrod et al. 2008). Here we reconsider these processes and motivated by the observations of Martín et al. (2008), construct a model for a hot core including both gas phase and grain surface formation and destruction of HNCO. We set out to model both the $\mathrm{HNCO}$ abundance and the HNCO/CS ratio as a function of time for such a region. We also aim to explore the response of the gas-phase formation of HNCO to changing physical conditions. Besides modelling HNCO in hot cores, simple models of a dark cloud and PDRs are also presented for comparison.

\section{Model descriptions}

Our hot core model consists of two distinct stages. The first stage is the collapsing cloud (CC) phase which follows the isothermal collapse of a dark molecular cloud whilst the second stage follows the evolution at constant density and a specified temperature - this is the HC phase. Along with the CC and HC models simple dark cloud (DC) and PDR models were also produced. These models were similar to the HC models in that no collapse was included but different physical conditions were used. All the models described in this paper were single point models.

\subsection{Physical model}

The CC model begins at a time $t=0$ with an homogeneous dark molecular cloud at a temperature, $T$ and an initial total hydrogen number density of $2 \times 10^{4} \mathrm{~cm}^{-3}$. The total hydrogen number density is defined as $n_{\mathrm{H}}=2 n\left(\mathrm{H}_{2}\right)+n(\mathrm{H})$ and is equal to the cloud's density. It was assumed that the cloud was shielded by a visual extinction, $A_{\mathrm{v}}$, of 15 magnitudes and the standard Galactic UV radiation field and cosmic ray ionisation rates were adopted. These physical conditions were chosen to be representative of a typical Galactic molecular cloud (e.g. Millar \& Herbst 1990). The cloud was then allowed to collapse isothermally following the modified free-fall collapse formula (see Spitzer 1978);

$\frac{\mathrm{d} n(t)}{\mathrm{d} t}=B\left(\frac{n(t)^{4}}{n_{\mathrm{o}}}\right)^{1 / 3}\left\{24 \pi G m_{\mathrm{H}} n_{\mathrm{o}}\left[\left(\frac{n(t)}{n_{\mathrm{o}}}\right)^{1 / 3}-1\right]\right\}^{1 / 2} \mathrm{~cm}^{-3} \mathrm{~s}^{-1}$,

where $B$ is the retardation factor and a value of 0.7 was adopted following Rawlings et al. (1992), $n_{\mathrm{o}}$ is the initial cloud density (total hydrogen number density) prior to the collapse being initiated, $G$ is the Universal Gravitational constant and $m_{\mathrm{H}}$ is the atomic mass of hydrogen.

The collapse was halted once $n_{\mathrm{H}}=2 \times 10^{7} \mathrm{~cm}^{-3}$ which occurs after approximately $5 \times 10^{5}$ years. This density was chosen to be representative of a typical hot core. As soon as this density was reached the model terminated and this was considered to be the end of the CC stage. The final chemical states of these collapse models were used as the initial chemistry of the subsequent
Table 1. Summary of the seven collapsing cloud models.

\begin{tabular}{ccl}
\hline \hline Model & $T(\mathrm{~K})$ & HNCO formation chemistry \\
\hline CC1 & 10 & All \\
CC2 & 10 & Gas phase only \\
CC3 & 10 & Surface only (reactions a,b) \\
CC4 & 10 & All, reduced sulphur \\
CC5 & 20 & All \\
CC6 & 50 & All \\
CC7 & 100 & All \\
\hline
\end{tabular}

Notes. $T$ is the isothermal temperature of the collapse.

hot core phases. A summary of the physical parameters adopted for the seven CC models used in this work is given in Table 1.

The HC model represents the second stage of the overall evolution. The HC was considered to be an homogeneous cloud at the final density reached at the end of the collapse phase, $n_{\mathrm{H}}=2 \times 10^{7} \mathrm{~cm}^{-3}$, in which the $A_{\mathrm{v}}$ was high enough that the influence of external FUV photons is negligible. For the duration of the $\mathrm{HC}$ model these physical conditions remained fixed.

A total of $10 \mathrm{HC}$ models were produced. Models 1 through 4 all had the same physical conditions including a temperature of $200 \mathrm{~K}$, meant to represent the heating by a newly formed central star. The four models differ in their chemical networks. On the other hand, models 5 to 10 all had the same chemistry but differing temperatures (the temperature of the initial the collapsing clouds for these models also differed). A summary of these models can be seen in Table 2

In order to follow chemistry on dust surfaces it was necessary to include dust grains in the models. By assuming a gas mass to dust mass ratio of 100 , the dust number density (relative to $\left.n_{\mathrm{H}}\right)$ was calculated to be $1.33 \times 10^{-12}$. A single population of spherical dust grains was then assumed with each grain having a radius of $10^{-5} \mathrm{~cm}$. The total number of binding sites on each grain was approximately $10^{6}$ assuming a surface density of $7.9 \times$ $10^{14} \mathrm{~cm}^{-2}$.

The DC and PDR models were modelled in a similar fashion to the HC. The physical conditions were kept constant in each case and no collapse was included. The DC was modelled using a $T=10 \mathrm{~K}, n_{\mathrm{H}}=2 \times 10^{4} \mathrm{~cm}^{-3}$ and an $A_{\mathrm{v}}=15$, whilst the PDR model adopted conditions representative of the Horsehead nebula (Habart et al. 2005); $T=50 \mathrm{~K}, n_{\mathrm{H}}=2 \times 10^{4} \mathrm{~cm}^{-3}, A_{\mathrm{v}}=1$ and an enhanced FUV radiation field equal to 60 times the interstellar value as used in all the other models. In order to mimic the effects of self-shielding of $\mathrm{H}_{2}$ and $\mathrm{CO}$, which is not explicitly included in the code, the photodissociation rates for both these species were set to zero in the PDR models. All the DC and PDR models produced in this work are also summarised in Table 2. A range of PDR models were run to demonstrate the effect of different aspects of the HNCO chemistry, different densities and different initial chemical compositions, either atomic or evolved from a collapsing cloud. A very dense PDR with an initially evolved chemistry such as models PDR4 and PDR5 were explored to represent clumps exposed to the UV radiation from a very nearby young massive star.

\subsection{Chemical model}

For the CC and DC models, along with PDR1, PDR2 and PDR3, all species were initially present in their elemental (atomic) form. The only exception was hydrogen which was assumed to be predominantly molecular with only a small fraction present 
D. M. Tideswell et al.: The abundance of $\mathrm{HNCO}$ and its use as a diagnostic of environment

Table 2. Summary of the dark cloud (DC), hot core (HC) and PDR models used to model the time dependent HNCO gas-phase abundance.

\begin{tabular}{cccll}
\hline \hline Model & $T(\mathrm{~K})$ & $n_{\mathrm{H}}\left(\mathrm{cm}^{-3}\right)$ & Initial chemistry & HNCO formation chemistry \\
\hline DC1 & 10 & $2.0 \times 10^{4}$ & Atomic & Reaction c only \\
DC2 & 10 & $2.0 \times 10^{4}$ & Atomic & Reactions c,d,e and f \\
DC3 & 10 & $2.0 \times 10^{4}$ & Atomic & Reactions d,e, f and g \\
DC4 & 10 & $2.0 \times 10^{4}$ & Atomic & Reactions c,d,e,f and g \\
HC1 & 200 & $2.0 \times 10^{7}$ & CC1 & All \\
HC2 & 200 & $2.0 \times 10^{7}$ & CC2 & Gas phase only \\
HC3 & 200 & $2.0 \times 10^{7}$ & CC3 & Surface only (reactions a,b) \\
HC4 & 200 & $2.0 \times 10^{7}$ & CC4 & All \\
HC5 & 20 & $2.0 \times 10^{7}$ & CC5 & All \\
HC6 & 20 & $2.0 \times 10^{7}$ & CC1 & All \\
HC7 & 50 & $2.0 \times 10^{7}$ & CC6 & All \\
HC8 & 50 & $2.0 \times 10^{7}$ & CC1 & All \\
HC9 & 100 & $2.0 \times 10^{7}$ & CC7 & All \\
HC10 & 100 & $2.0 \times 10^{7}$ & CC1 & All \\
PDR1 & 50 & $2.0 \times 10^{4}$ & Atomic & All \\
PDR2 & 50 & $2.0 \times 10^{4}$ & Atomic & Gas phase only \\
PDR3 & 50 & $2.0 \times 10^{4}$ & Atomic & Surface only (reactions a,b) \\
PDR4 & 50 & $2.0 \times 10^{7}$ & CC1 & All \\
PDR5 & 50 & $2.4 \times 10^{5}$ & CC1 & All \\
\hline
\end{tabular}

Notes. $T$ is the gas-phase temperature and $n_{\mathrm{H}}$ is the total hydrogen number density (both held constant). The initial chemistry was either atomic, where all species (except $\mathrm{H}$ ) were in their elemental form, or evolved, where abundances from a collapsed cloud (CC) model were used. The collapsing cloud models are listed in a separate table.

Table 3. Standard initial chemical abundances relative to the total hydrogen number density.

\begin{tabular}{lclclc}
\hline \hline & $Y(\mathrm{X})$ & & $Y(\mathrm{X})$ & & $Y(\mathrm{X})$ \\
\hline $\mathrm{H}_{2}$ & 0.5 & $\mathrm{H}$ & $1.00 \times 10^{-6}$ & $\mathrm{He}$ & 0.1 \\
$\mathrm{C}$ & $1.32 \times 10^{-4}$ & $\mathrm{~N}$ & $7.50 \times 10^{-5}$ & $\mathrm{O}$ & $3.19 \times 10^{-4}$ \\
$\mathrm{~S}$ & $1.86 \times 10^{-5}$ & $\mathrm{Si}^{+}$ & $2.46 \times 10^{-7}$ & $\mathrm{Fe}^{+}$ & $1.50 \times 10^{-8}$ \\
\hline
\end{tabular}

Notes. $Y(\mathrm{X})=n(\mathrm{X}) / n_{\mathrm{H}}$.

Table 4. Key reactions included in the chemical network relevant to the formation of HNCO.

\begin{tabular}{crlll}
\hline \hline Label & \multicolumn{3}{c}{ Reaction } & Reference \\
\hline a & GOCN + GH & $\rightarrow$ & GHNCO & AR77 \\
b & GNH + GCO & $\rightarrow$ & GHNCO & GWH08 \\
c & OCN + H & $\rightarrow$ & HNCO + H & TTH99 \\
d & $\mathrm{H}_{2} \mathrm{NCO}^{+}+\mathrm{e}^{-}$ & $\rightarrow$ & $\mathrm{HNCO}_{+} \mathrm{H}$ & I77 \\
e & $\mathrm{H}_{2}+\mathrm{OCN}^{+}$ & $\rightarrow$ & $\mathrm{HNCO}^{+}+\mathrm{H}$ & $\mathrm{I} 77$ \\
$\mathrm{f}$ & $\mathrm{H}_{2}+\mathrm{HNCO}^{+}$ & $\rightarrow$ & $\mathrm{H}_{2} \mathrm{NCO}^{+}+\mathrm{H}$ & $\mathrm{I} 77$ \\
$\mathrm{~g}$ & $\mathrm{He}^{+}+\mathrm{OCN}$ & $\rightarrow$ & $\mathrm{He}+\mathrm{OCN}^{+}$ & - \\
\hline
\end{tabular}

Notes. The prefix G denotes surface species. AR77; Allen \& Robinson (1977), GWH08; Garrod et al. (2008), TTH99; Turner et al. (1999), I77; Iglesias (1977).

as atoms. These initial abundances (relative to $n_{\mathrm{H}}$ ) are given in Table 3. For the HC models, along with PDR4 and PDR5, an evolved initial chemistry was used. The initial abundances at the start of these models used the abundances taken from a CC model. All the HC models and PDR4 used abundances taken from a $\mathrm{CC}$ at the final density, $2 \times 10^{7} \mathrm{~cm}^{-3}$, whereas PDR5 used abundances from a CC model at an earlier stage in the collapse. In this case the density reached in the collapse was equal $2.4 \times 10^{5} \mathrm{~cm}^{-3}$.

For all the models, once the chemistry had been initialised, the subsequent evolution of all species was followed via a chemical reaction network. This network included both reactions in the gas phase and on dust grain surfaces, along with the coupling mechanisms of accretion and thermal desorption only.

The gas-phase chemistry included all the reactions from the latest UMIST database for astrochemistry (UDfA ${ }^{1}$ ), Rate06 (Woodall et al. 2007). For the grain-surface chemistry over 200 reactions were included from Allen \& Robinson (1977), Hasegawa et al. (1992) and Hasegawa \& Herbst (1993). Reactions on the surface were treated using the modified rate equation method as used by Ruffle \& Herbst (2000).

In order to more completely model the HNCO gas-phase abundance some additional chemistry was included. Five surface reactions involving $\mathrm{HNCO}$ were taken from the expanded OSU gas-grain reaction network (Garrod et al. 2008) comprising of a single formation path (reaction b in Table 4) plus four destruction mechanisms. This surface chemistry was complemented by some gas phase reactions (also from Garrod et al. 2008) which followed the destruction of four new species (HNCHO, $\mathrm{HNCOCHO}, \mathrm{HNCONH}$ and $\mathrm{HNCOOH}$ ) formed from surface processing of HNCO. The destruction of $\mathrm{HNCO}$ in the gas phase was also included. This followed the treatment of Turner et al. (1999) and included the reactions between HNCO and the following species; $\mathrm{H}^{+}, \mathrm{H}_{3}^{+}, \mathrm{He}^{+}, \mathrm{C}^{+}, \mathrm{H}_{3} \mathrm{O}^{+}, \mathrm{CO}^{+}, \mathrm{HCO}^{+}$, $\mathrm{OCN}^{+}$and $\mathrm{HNCO}^{+}$. The rate coefficients for these reactions were taken from the ion-neutral reaction database compiled by Anicich (2004). The photodestruction of HNCO by FUV photons and cosmic ray induced photodissociation was also incorporated (Gredel et al. 1989; Roberge et al. 1991). Reactions with ions, particularly $\mathrm{H}_{3}^{+}$and $\mathrm{He}^{+}$dominate the destruction of HNCO in both dark clouds and hot cores.

Two gas-phase formation reactions for $\mathrm{HNCO}$ were added (reactions $\mathrm{c}$ and $\mathrm{d}$ in Table 4). The first reaction (c) is a neutralneutral reaction whilst the second (d) is the dissociative recombination of $\mathrm{H}_{2} \mathrm{NCO}^{+}$. For reaction $\mathrm{c}$ the rate coefficient is poorly known. Turner et al. (1999) adopted a value of $10^{-13} \mathrm{~cm}^{3} \mathrm{~s}^{-1}$ in their dark cloud model. However the energy barrier for this reaction may be much larger than suggested by this number and as

\footnotetext{
${ }^{1}$ UDfA; www . udfa.net
} 
such this reaction may only become significant in regions much warmer than dark clouds. The rate coefficient for this reaction has been measured but only at temperatures above $300 \mathrm{~K}$ (see the NIST chemical kinetics database ${ }^{2}$ ). A fit to the available data provides an expression for the temperature dependent rate coefficient;

$k(T)=1.5 \times 10^{-11} \times \exp \left(\frac{-4465}{T}\right) \mathrm{cm}^{3} \mathrm{~s}^{-1}$,

where $T$ is the temperature. At $10 \mathrm{~K}$ this gives a considerably smaller value than that used by Turner et al. (1999). We used model DC1 to examine how the rate of reaction $\mathrm{c}$ affects the HNCO abundance - the model was run several times, each time using a different value for the rate coefficient ranging from $10^{-22} \mathrm{~cm}^{3} \mathrm{~s}^{-1}$ to $10^{-8} \mathrm{~cm}^{3} \mathrm{~s}^{-1}$ (increasing the value by two orders of magnitude each time). For all other models in which reaction $\mathrm{c}$ was included the rate was calculated using Eq. (2).

Three further reactions were added that form "parent" species needed for the formation of HNCO. Reactions e and $f$ are ion-neutral reactions that are the first two reactions in a three stage process. The actual formation of HNCO in this reaction channel is via reaction d (Iglesias 1977). The rates of these reactions are calculated using the Langevin rate. The final reaction $(\mathrm{g})$ is a charge exchange reaction between $\mathrm{He}^{+}$and OCN which produces $\mathrm{OCN}^{+}$. This reaction represents a third, new, reaction channel between these two species since the UDfA already contains two reactions between $\mathrm{He}^{+}$and $\mathrm{OCN}$. A rate coefficient of $10^{-9} \mathrm{~cm}^{3} \mathrm{~s}^{-1}$ was adopted for reaction $\mathrm{g}-$ representative of charge exchange reactions.

All models were allowed to evolve chemically for $10^{7}$ years, except for the collapsing cloud models which were stopped once the final density was reached.

\section{Results}

\subsection{Gas phase formation of HNCO in dark clouds}

For the first part of our investigation, using DC1, we explored the effect the rate coefficient for reaction $\mathrm{c}$ has on the abundance of HNCO in the gas phase. Figure 1 shows the HNCO abundance along with that of OCN for a range assumed rate coefficients for reaction c. For a rate coefficient of $10^{-18} \mathrm{~cm}^{3} \mathrm{~s}^{-1}$ and above it is possible to achieve HNCO abundances within the observed range of $1.4 \times 10^{-10}$ to $5.4 \times 10^{-8}$ (MacDonald et al. 1996; Bisschop et al. 2007). Equation (2) implies that for the $\sim 4500 \mathrm{~K}$ barrier which laboratory data (NIST) infer for this reaction, temperatures above $277 \mathrm{~K}$ are required to reach this value of the rate coefficient for reaction c. Alternatively for this reaction to be effective at a gas temperature of $10 \mathrm{~K}$, the barrier would have to be less than $\sim 165 \mathrm{~K}$, much lower than measurements indicate. In other words, at the temperatures of dark molecular clouds these reactions do not occur.

Model DC2 illustrates how the other gas-phase formation route (via reaction d) influences the $\mathrm{HNCO}$ abundance. Reaction $\mathrm{c}$ was also included in this model and the rate was varied as before. Figure 2 shows the HNCO and OCN abundances along with those for $\mathrm{OCN}^{+}$. A lower limit for the fractional HNCO abundance (at steady state) is effectively set at $1.1 \times 10^{-11}$. This corresponds to the DC2 model with a rate cofficient of 0 for reaction $\mathrm{c}$ (the solid red line in Fig. 2). In this model HNCO is underabundant compared to observations by

\footnotetext{
2 The NIST chemical kinetics database; http://kinetics.nist. gov/kinetics/index. jsp
}
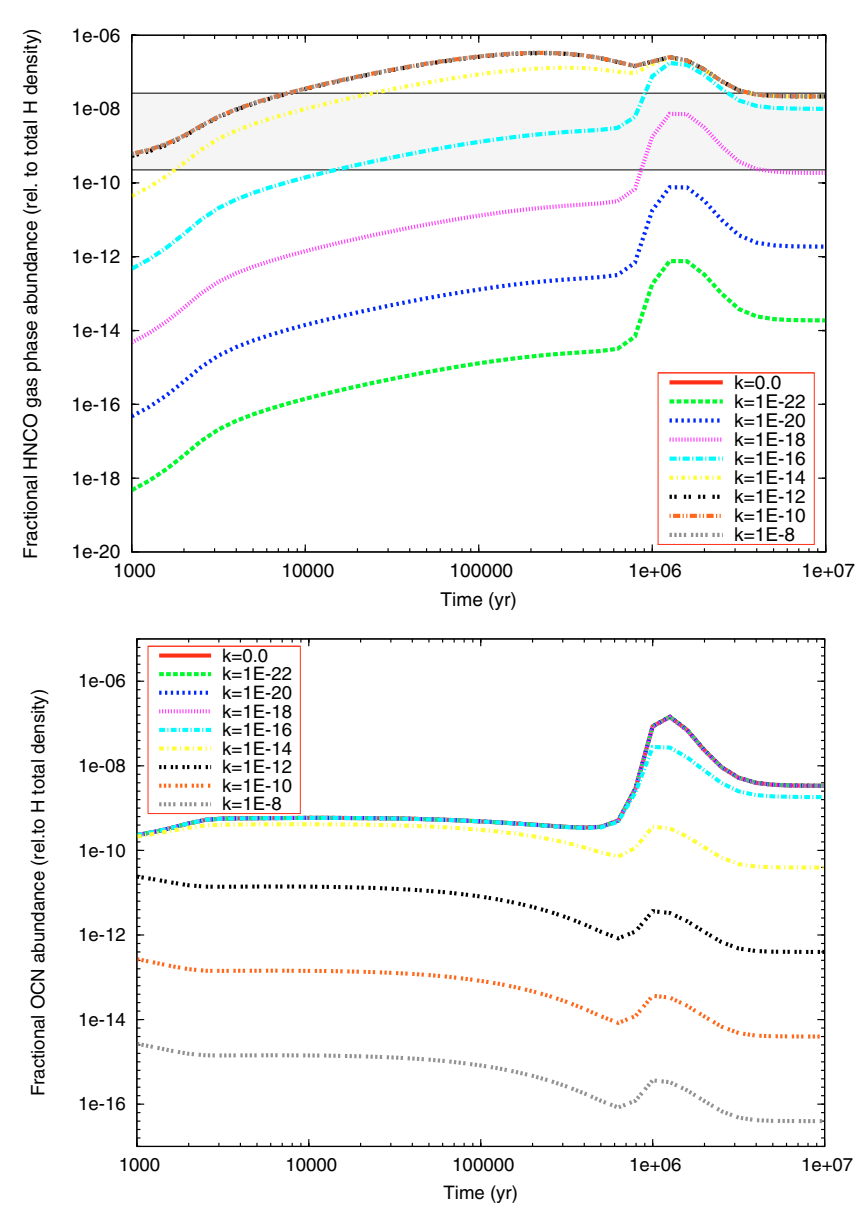

Fig. 1. Time dependent, gas phase relative abundances for HNCO (top) and OCN (bottom) as a function of rate coefficient for reaction $\mathrm{c}$ in a non-collapsing dark cloud (model DC1). All other HNCO formation pathways are prohibited, both in the gas-phase and on grain surfaces. Reaction $g$ has rate coefficient of 0.0 . The shaded region defines the range of values for the observed $\mathrm{HNCO}$ abundance, $1.4 \times 10^{-10}$ to $5.4 \times$ $10^{-8}$.

over 2 orders of magnitude. However, as seen for DC1, when the rate coefficient for reaction c increases, it is possible to achieve higher abundances. There is however very little difference between DC1 and DC2 for those models with non-zero rate coefficients for reaction c. Figure 3 shows the gas-phase abundance for the key reactants/products involved in the gas-phase production of HNCO (model DC3). This shows that there is OCN in the gas-phase but due to the low temperature this is not converted into HNCO. Instead, at low temperatures HNCO formation through reaction d (via reactions e and f) clearly dominates. The fourth and final DC model, DC4, includes all the gas phase formation reactions listed in Table 4 (but in common with the other DC models, no grain surface processes) and the reaction rate for reaction $\mathrm{c}$ has been varied. The abundance profiles for this model are seen in Fig. 4. For small values of the rate coefficient of reaction $\mathrm{c}$, the inclusion of reaction $\mathrm{g}$ increases the HNCO abundance. However, to achieve HNCO abundances in the observable range for ages less than $10^{6}$ years, the Turner rate must be greater than $10^{-16} \mathrm{~cm}^{3} \mathrm{~s}^{-1}$, which would imply temperatures of $>370 \mathrm{~K}$. At ages greater than $\sim 10^{6}$ years reaction $g$ appears to be able to produce sufficient HNCO to match the observations even for a much reduced rate for reaction c (indeed even a rate of zero). However interpreting this late time rise in $\mathrm{HNCO}$ abundance needs to be treated with caution as these models only 
D. M. Tideswell et al.: The abundance of $\mathrm{HNCO}$ and its use as a diagnostic of environment
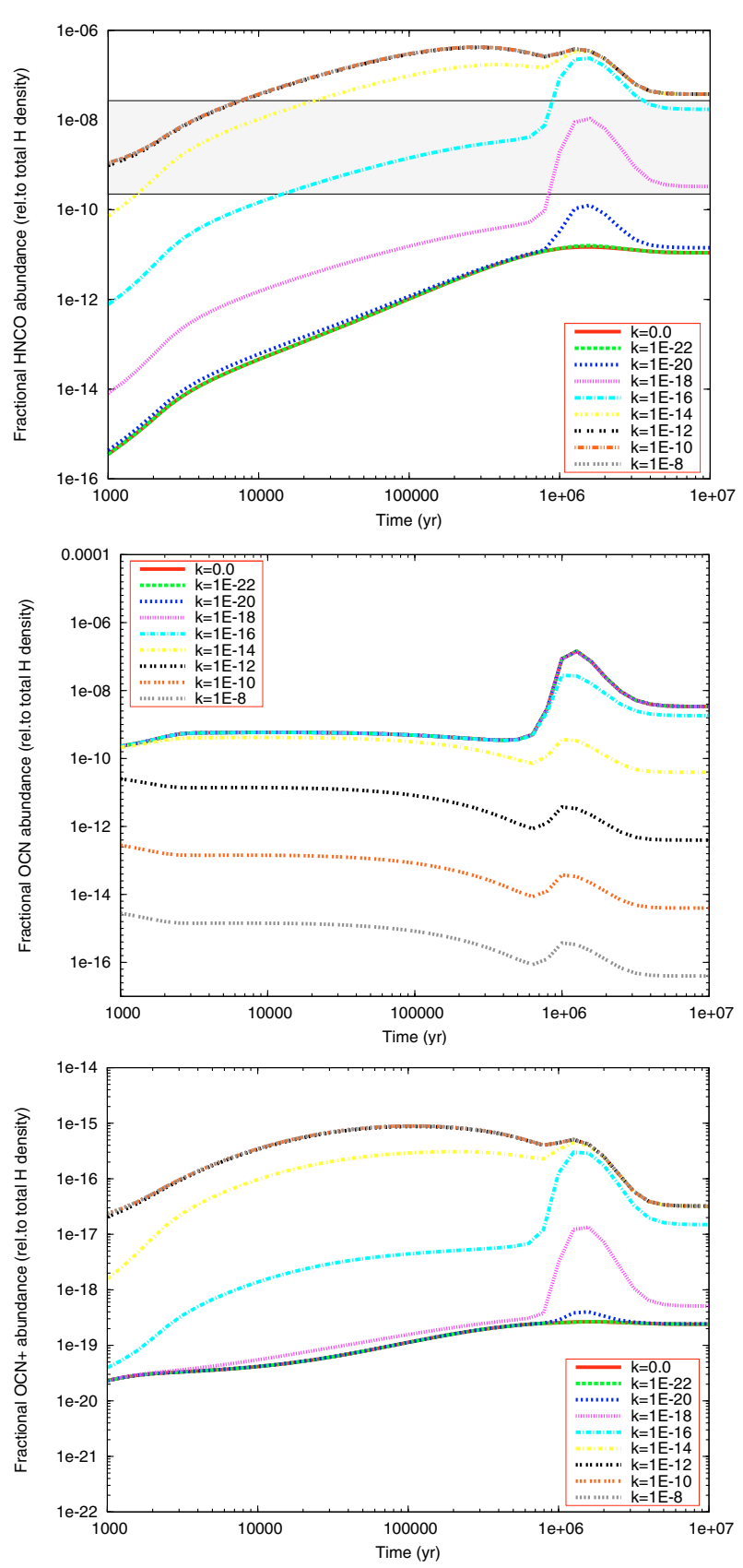

Fig. 2. Time dependent, gas phase relative abundances for HNCO (top), OCN (middle) and $\mathrm{OCN}^{+}$(bottom) as a function of rate coefficient for reaction $\mathrm{c}$ in a non-collapsing dark cloud (model DC2). All gasphase HNCO formation pathways are allowed; grain-surface reactions are prohibited. Reaction $g$ has rate coefficient of 0.0 . The shaded region defines the range of values for the observed $\mathrm{HNCO}$ abundance

include gas phase reactions and so do not include the effect on the gas phase abundance of freeze-out on to the grains which will be very significant at these late times. This peak in HNCO abundance might occur earlier in models of denser clouds, but the effect of freeze-out on the gas phase abundances would also occur earlier.

\subsection{HNCO in photodissociation regions}

Figure 5 shows the results for our PDR models. The HNCO abundances in PDR regions are even lower than those found in DC. For the three PDR models with an initially atomic chemistry

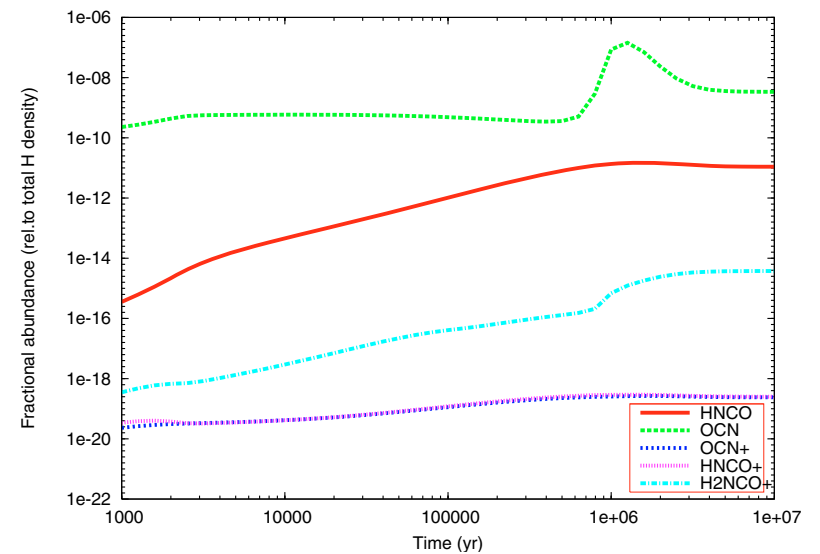

Fig. 3. Time dependent, gas phase relative abundances for various species in a non-collapsing dark cloud using only reactions $\mathrm{d}$, e and f to produce HNCO (model DC3).

(PDR1, PDR2 and PDR3) the largest late time abundance of HNCO $\left(10^{-17}\right)$ is produced in the models with gas phase formation (PDR1 and PDR2), but these produce up to 9 orders of magnitude less HNCO than in a DC model. In model PDR3, which allows only surface reactions, HNCO is even less abundant, reaching a maximum value of $10^{-20}$. The final two PDR models are also presented in Fig. 5. Both models follow a similar trend, that is the HNCO abundance is steadily decreasing with time, reaching a plateau at later times. However, the HNCO abundance in PDR4 is initially larger by about two orders of magnitude than in PDR5, although they ultimately reach similar abundances of about $2 \times 10^{-18}$. None of the PDR models ever approach the abundances of HNCO seen in DCs and HCs - even for the PDR models which start with previously evolved chemistries (PDR4 and PDR5).

The warmer conditions in these PDR models, largely due to the reduced optical depth and enhanced radiation field resulting in the heating of dust grains, lead to faster desorption meaning that once formed HNCO is never on the grains for long (at the $50 \mathrm{~K}$ used in these models the surface lifetime is approximately 100 years) so surface formation is limited. Furthermore, HNCO is efficiently destroyed by both FUV and cosmic ray induced photodestruction reactions. At no time during the evolution of any of our PDR models does the HNCO abundance come close to the $\sim 10^{-9}$ observed by Martín et al. (2008) towards PDR regions. As a consequence of this low HNCO abundance, the ratio of the abundance of HNCO to the CS abundance in these PDR models is also many orders of magnitude lower than seen by Martín et al. (2008) (Fig. 7). The origin of the HNCO observed by Martín et al. (2008) apparently in PDRs is therefore unclear, although it may be related to shielding of the molecular gas.

\subsection{HNCO in hot cores}

Figure 6 shows the HNCO gas-phase abundance as a function of time for all the hot core models 1 to 10 . Only times between 1000 years to $1 \mathrm{Myr}$ (since the HC's switch-on) are plotted as HCs are transient objects and are relatively short-lived phases of high-mass star formation lasting less than $1 \mathrm{Myr}$.

$\mathrm{HC} 1$ includes the complete reaction network described earlier. The HNCO abundance for this model does reached observed levels. Beyond $\sim 10^{4}$ yrs values greater than $10^{-9}$ are seen and a peak of $\sim 2 \times 10^{-8}$ is reached at around 0.2-0.3 Myr. For the model with surface formation only (HC3) the lower observed 

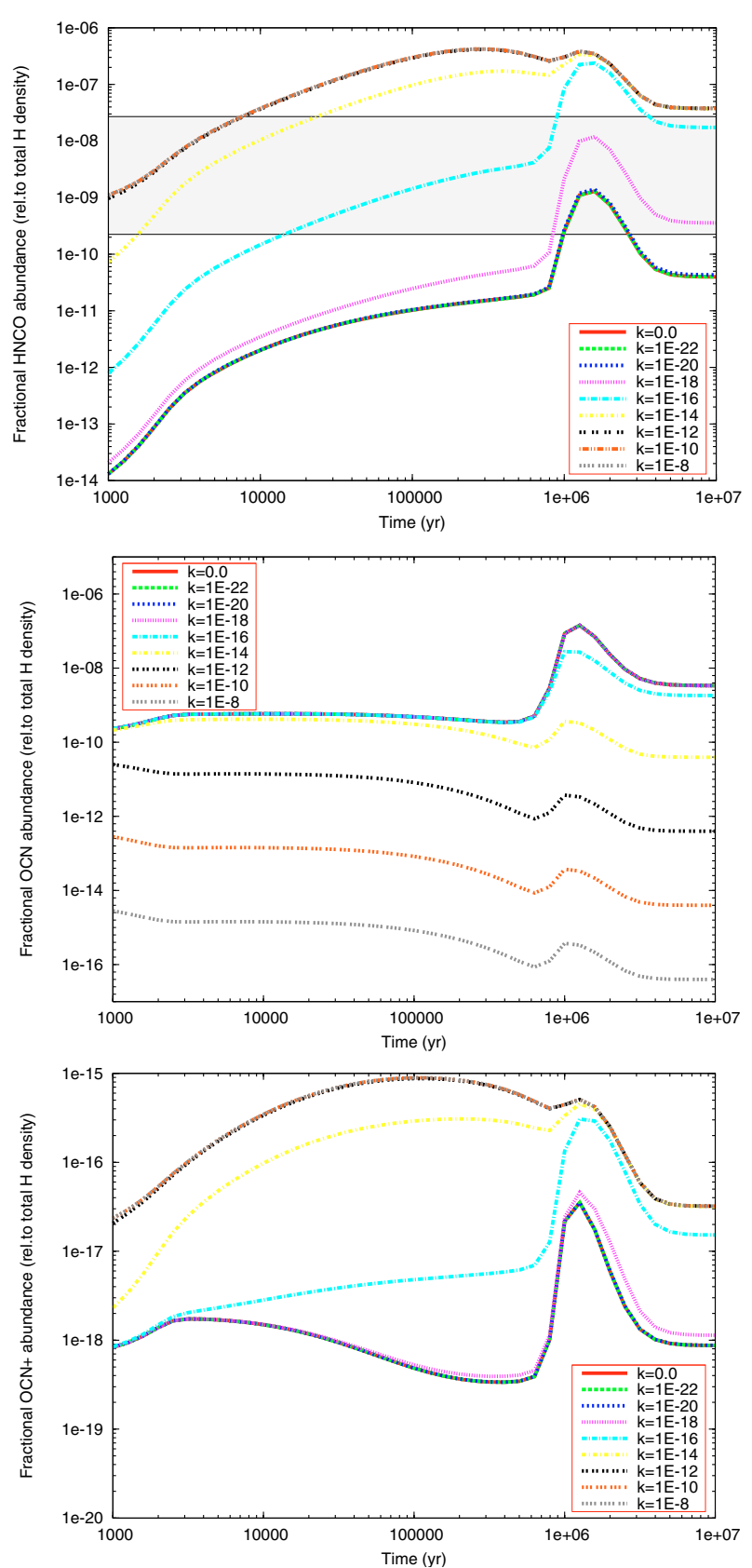

Fig. 4. Time dependent, gas phase relative abundances for $\mathrm{HNCO}$ (top), OCN (middle) and $\mathrm{OCN}^{+}$(bottom) as a function of rate coefficient for reaction $\mathrm{c}$ in a non-collapsing dark cloud (model DC4). All gas-phase $\mathrm{HNCO}$ formation pathways are allowed but grain-surface reactions are prohibited. Reaction $\mathrm{g}$ has rate coefficient of $1.0 \times 10^{-9} \mathrm{~cm}^{3} \mathrm{~s}^{-1}$. The shaded region defines the range of values for the observed HNCO abundance

limit for $\mathrm{HNCO}$ is reached but only beyond $10^{5}$ yr. The HNCO abundance in $\mathrm{HC} 4$ (which differs from $\mathrm{HC} 1$ only in the elemental sulphur abundance) is almost identical in behaviour as HC1. The major differences are that higher abundances are reached at slightly earlier times and the peak value of just over $2 \times 10^{-8}$ remains for a further $0.1 \mathrm{Myr}$.

The gas-phase formation only $\mathrm{HC}$ model (HC2) shows that the abundance for HNCO remains relatively flat and never exceeds a few $10^{-11}$, and even then only at HC ages greater than $0.3 \mathrm{Myr}$. As discussed above, this indicates that even the relatively high temperatures in hot cores, the gas-phase reactions by

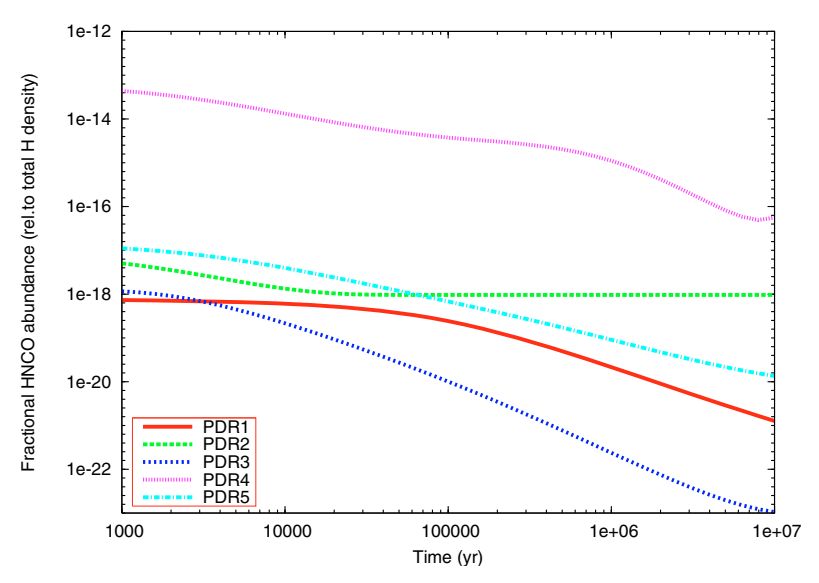

Fig. 5. Time dependent, relative gas phase abundance of HNCO for the five PDR models. For comparison the observed value is between $1.4 \times 10^{-10}$ abd $5.4 \times 10^{-9}$. Note that curves for PDR1 and PDR2 are coincident.
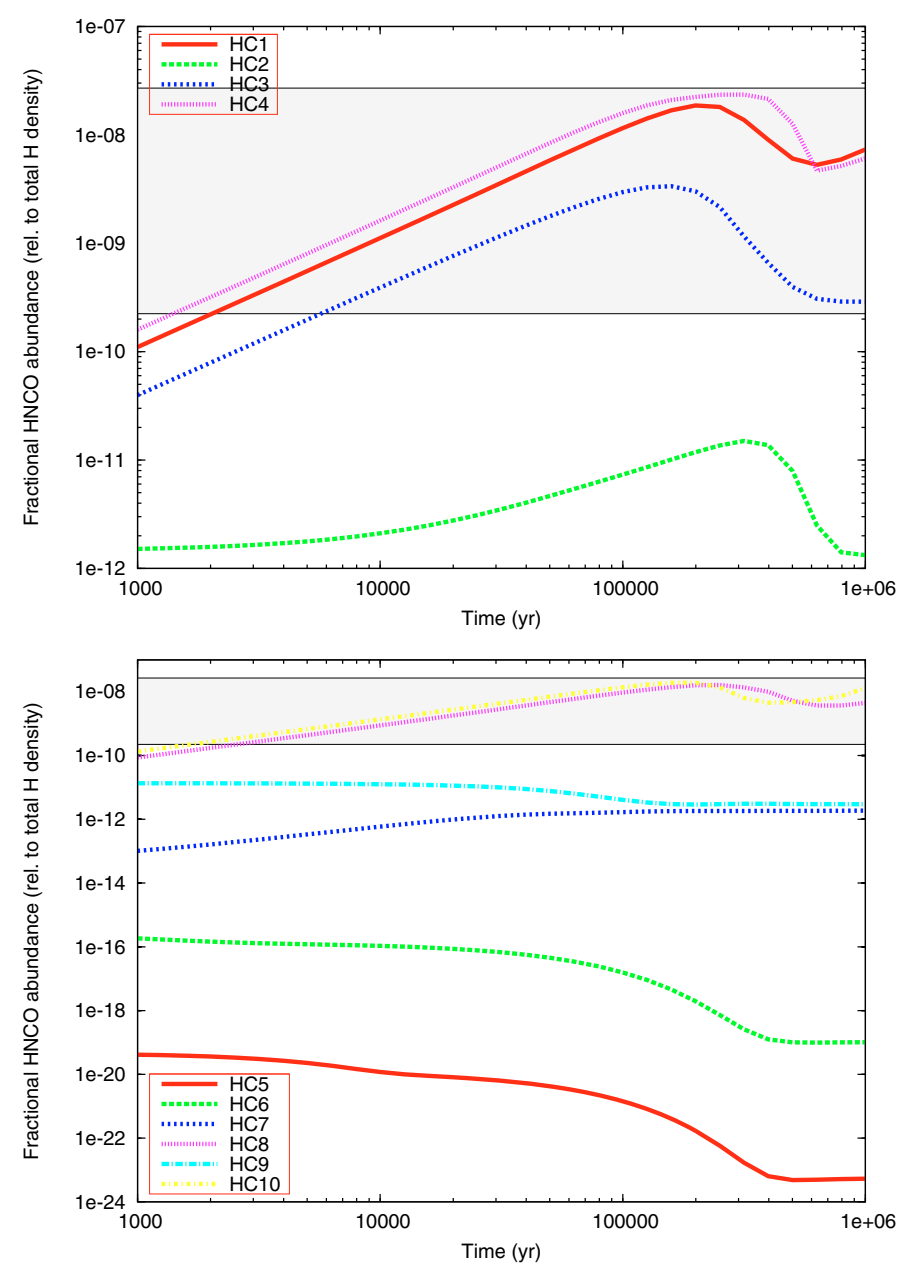

Fig. 6. Relative HNCO gas-phase abundance as a function of time for hot core models (HC) 1-4 (top) and 5-10 (bottom).

themselves are incapable of producing $\mathrm{HNCO}$ at observed levels.

The remaining HC models all had the same chemical networks (the full network with all reactions "on"), it was only the initial and final temperatures that differed. In fact for the sake of comparison three of the models, HC5, HC7 and HC9, each had a constant temperature during the initial collapse and 
throughout subsequent constant density evolution. The first two models, HC5 and HC6, had the same post-collapse temperature (20 K), however HC6 used evolved abundances from a cloud collapsed at $10 \mathrm{~K}$ where as HC5 used abundances from a cloud at $20 \mathrm{~K}$. HC5 never has a fractional HNCO abundance above $10^{-19}$, whilst HC6 never really exceeds $10^{-16}$. HC6 has consistently more $\mathrm{HNCO}$ throughout - due to a lower temperature collapse phase temperature. At lower temperatures the rate of thermal desoprtion is reduced such that the surface chemistry becomes more efficient. This means there is more $\mathrm{NH}, \mathrm{CO}$ and OCN on grain surfaces - the key reactants for the surface formation of HNCO. For the models presented in this paper, the surface reaction between $\mathrm{OCN}$ and $\mathrm{H}$ is more important for the formation of $\mathrm{HNCO}$ than the reaction between $\mathrm{CO}$ and $\mathrm{NH}$.

The next two models had post-collapse temperatures of $50 \mathrm{~K}$. Again, the only difference being the collapse temperature (HC7 with $T=50 \mathrm{~K}, \mathrm{HC} 8$ with $T=10 \mathrm{~K}$ ). Both of these models have higher HNCO abundances compared to HC5 and HC6. Model $\mathrm{HC} 8$ is almost exactly the same as $\mathrm{HC} 1$, but $\mathrm{HC} 7$ has a relatively constant fractional abundance of around $10^{-12}$. Model HC9 had a collapse and post-collapse temperature of $100 \mathrm{~K}$. In this model the abundance of HNCO is roughly constant. At early times abundances just greater than $10^{-11}$ are seen, falling slowly to just above $10^{-12}$ beyond $0.1 \mathrm{Myr}$. The final model, HC10, also has a post-collapse temperature of $100 \mathrm{~K}$ but the collapse temperature is $10 \mathrm{~K}$. This has an almost identical abundance profile as HC8. These results indicate that in order to achieve HNCO gas-phase abundances similar to those observed, post-collapse temperatures greater than $50 \mathrm{~K}$ are required.

\subsection{The abundance of CS}

Our models can also be used to investigate the abundance of $\mathrm{CS}$ as function of time in hot cores (and PDRs). A detailed discussion of the important pathways for the evolution of sulphurbearing species, including CS, is given in Millar \& Herbst (1990). As mentioned above, CS is often used as a tracer of dense gas associated with star formation and Martín et al. (2008) suggested the use of the ratio of the abundance of HNCO to that of CS as measure of the importance of UV radiation in a region.

Figures 7 and 8 show the evolution of the abundance of CS in our PDR and HC models respectively. All three PDR models which start with atomic compositions (PDR1, PDR2 and PDR3) show identical behaviour. From its initial value of $2 \times 10^{-10}$ the abundance of CS monotomically decreases by a factor of $>10$ over the period covered by the model. For PDR 4 and PDR5 which start with evolved chemistries the CS abundance also monotomically decreases from a peak at early times, becoming approximately constant at later times. The highest abundance of CS in these models occurs are very early times in PDR5, where the peak abundance is $\sim 10^{-9}$. In the HC models the CS abundance is initially relatively constant, rising to a peak after about $10^{5} \mathrm{yr}$ before rapidly declining thereafter. Comparing models $\mathrm{HC} 1$ and $\mathrm{HC} 4$, which has a sulphur abundance ten times smaller that the other models, shows that the CS abundance throughout most the evolution of the HC scales nearly directly with the initial sulphur abundance.

\section{Discussion}

Hot core models $\mathrm{HC} 1, \mathrm{HC} 2$ and $\mathrm{HC} 3$ demonstrate that gas phase reactions alone can not produce a high enough abundance of HNCO to match observations. Reactions on the surfaces of

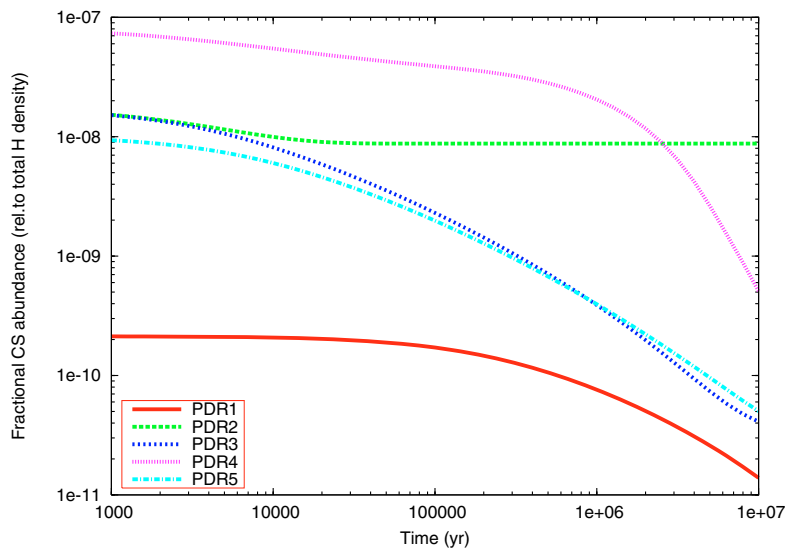

Fig. 7. Time dependent, relative gas phase abundance of CS for the five PDR models.

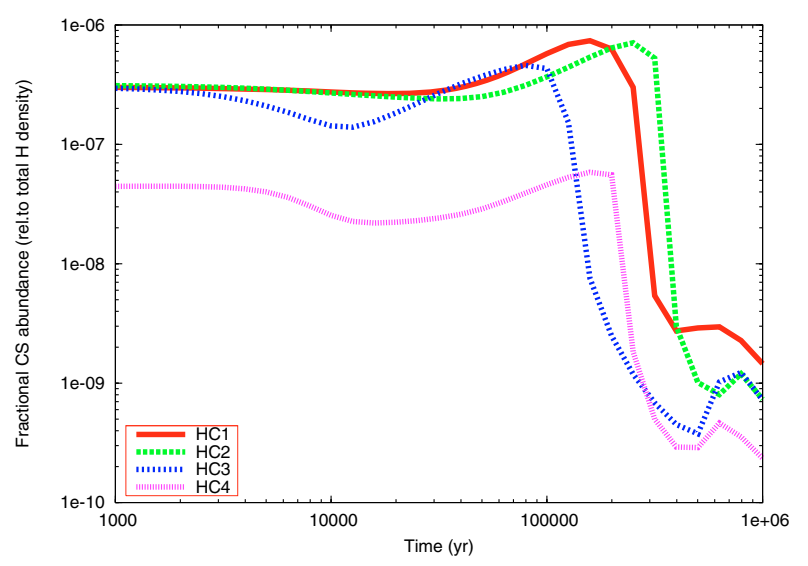

Fig. 8. Time dependent, relative gas phase abundance of CS for four hot core models (HC1-4).

grains are required. Of the models with a complete HNCO chemistry only models $\mathrm{HC1}, 8$ and 10 achieve observed HNCO abundances. (Model HC4 can also match the observed abundance, but only differs from $\mathrm{HC} 1$ in its initial sulphur adundance.) These models all have a low collapse temperature combined with high post-collapse (hot core) temperatures. It appears that during the collapse phase cooler temperatures are essential. (A HC model identical to $\mathrm{HC} 4$, but with an inital collapse phase at $20 \mathrm{~K}$, rather than $10 \mathrm{~K}$, produces a peak $\mathrm{HNCO}$ abundance of only $\sim 3 \times 10^{-14}$ ). The cold collapse allows accretion on to the surface of grains and hence surface production of HNCO. However, during the hot core phase, high temperatures are needed to allow the desorption of the surface species back into the gas-phase. The models with constant temperatures across both stages 1 and 2 of the hot core model are seemingly incapable of producing $\mathrm{HNCO}$ at observed levels.

Interestingly, although grain surface reactions are clearly the ultimate source of the HNCO, the HNCO abundance does not follow the expected time dependent behaviour of a typical parent species ejected from grain mantles. In the log-log figures presented here the relative HNCO abundance is seen to linearly increase with time, reaching a peak value of $\sim 10^{-8}$, consistent with the peak observed values, after about $2 \times 10^{5}$ years (Fig. 6). This growth suggests that the gas phase $\mathrm{HNCO}$ is in fact a daughter product from the species ejected from the grain mantles.

Examining the grain mantle abundances shows that although it is efficiently formed, $\mathrm{HNCO}$ is also rapidly processed to more 
complex species. It is the destruction of these species after their ejection which produces the gas phase HNCO. In our models the $\mathrm{HNCO}$ is allowed to form several daughter products ( $\mathrm{HNCHO}$, $\mathrm{HNCOCHO}, \mathrm{HNCONH}$ and $\mathrm{HNCOOH}$ ) on grain surfaces during the collapse phase. Once the hot core stage is reached it is these species that are ejected and subsequently destroyed in the gas-phase thus returning HNCO. Such a possibility has been previously suggested by Zinchenko et al. (2000), although these authors prefered the explanation that the HNCO they observed was formed in the gas phase in post-shock gas. If the HNCO in our models was not destroyed on the grains it would be returned to the gas-phase as soon as the temperature is increased to $200 \mathrm{~K}$ with a relative abundance of $\sim 2 \times 10^{-5}$. This abundance is several orders of magnitude overabundant compared to observations.

Based on its excitation temperature Bisschop et al. (2007) argue that HNCO is a "first generation" species ejected from grains. Our models indicate that this is not the case and suggest that this association of a molecular species with physical conditions on the basis of their excitation temperature alone can be misleading.

\subsection{HNCO/CS abundance ratio}

The HNCO/CS abundance ratio as a function of time is shown for the PDR models along with all the HC models in Fig. 9. The PDR models never reach values of the $\mathrm{HNCO} / \mathrm{CS}$ abundance ratio $>10^{-6}$, orders of magnitude smaller than observed. For model $\mathrm{HC} 1$ the ratio increases from about $2 \times 10^{-4}$ at $10^{3}$ years, peaking at $\sim 3$ around $3 \times 10^{5}$ years. Martín et al. (2008) observed ratios for $\mathrm{HNCO} /{ }^{13} \mathrm{CS}$ in the range $\sim 1$ to $\sim 100$. Adopting a value of 77 for the abundance ratio to $\mathrm{C} /{ }^{13} \mathrm{C}$ (Wilson \& Rood 1994), this would imply $\mathrm{HNCO} / \mathrm{CS}$ ratios of $\sim 0.013$ to $\sim 1.3$. The models span this ratio for times between approximately $10^{4}$ years to $2 \times 10^{5}$ years showing that the time dependent evolution of the chemistry of hot cores alone can produce the entire range of observed $\mathrm{HNCO} / \mathrm{CS}$ abundance ratios. Therefore, independent of its effect on the ratio, it is clear that $\mathrm{HNCO} / \mathrm{CS}$ is not a unique tracer of UV radiation.

As the figure also shows, the underlying sulphur abundance also has a direct effect on the absolute value of the HNCO to CS abundance ratio. When $\mathrm{S}$ is reduced by a factor of 10 , for a given time less than about few times $10^{5}$ years, the $\mathrm{HNCO} / \mathrm{CS}$ ratio increases by a factor of 10 . However for these times the evolution for different sulphur abundances is essentially independent of abundance. Overall the ratio monotonically increases with time up to a peak at a few times $10^{5}$ years. This generally monotonic behaviour suggests that for a given sulphur abundance this ratio could act as a "chemical clock" to constrain the ages of sources.

\section{Summary}

Our model shows that gas phase reactions appear incapable of producing $\mathrm{HNCO}$ in sufficient quantities (even at the $\sim 200 \mathrm{~K}$ temperature of hot cores) to match the observed abundance of this species. However the rate of a key gas phase reaction is uncertain and new laboratory determination of this rate coefficient is highly desirable. HNCO can be formed on icy surfaces by a variety of reactions. Despite the existence of these plausible mechanisms which can produce sufficient HNCO to match observations, laboratory data is again missing and should be obtained. Although it is produced on the grain surfaces in potentially large amounts, our models show that the gas phase $\mathrm{HNCO}$ is in fact a daughter product from the breakdown of more complex species to which the HNCO on the grains have been
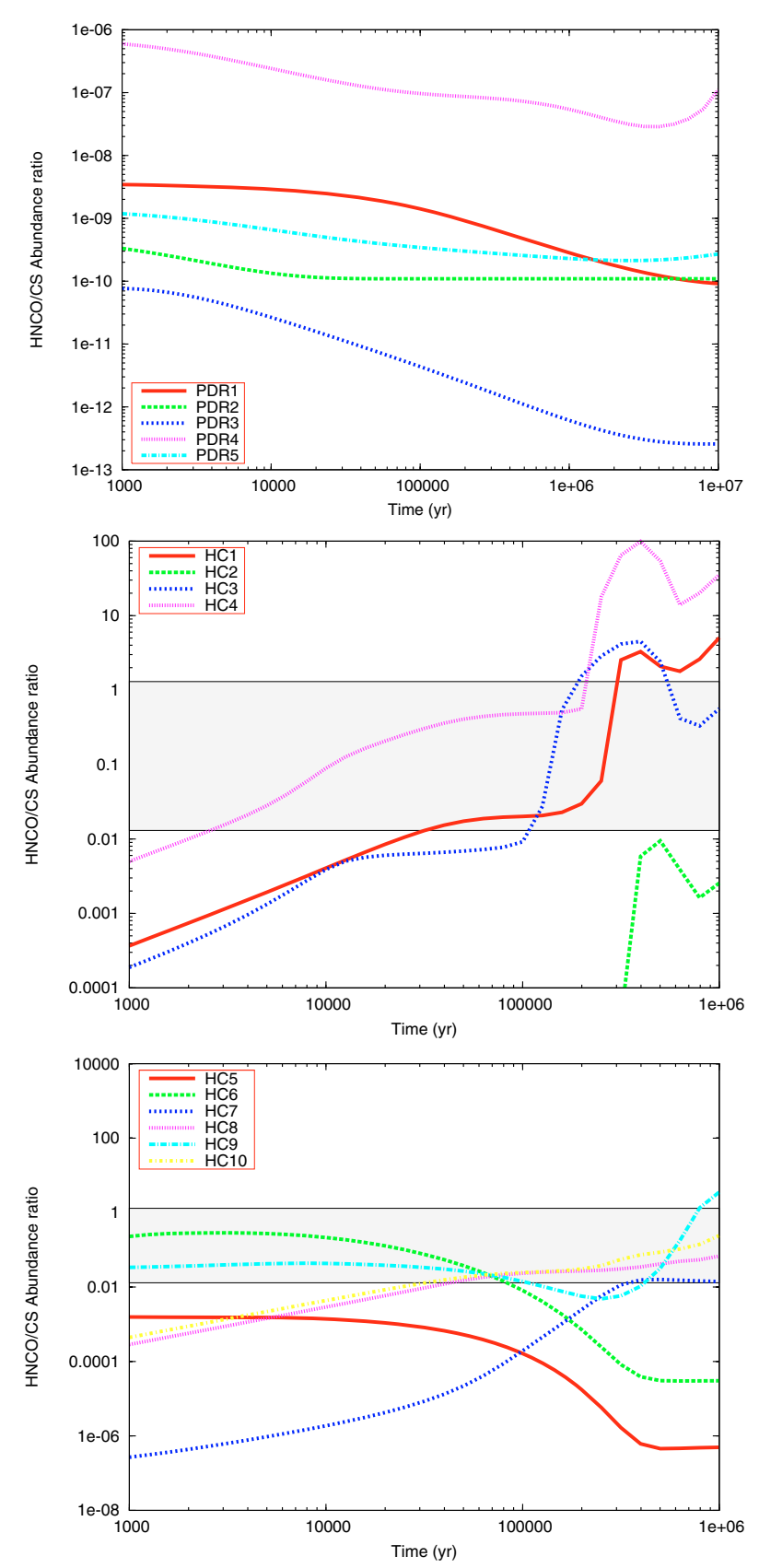

Fig. 9. HNCO/CS abundance ratio as a function of time for PDR models (top), hot core models 1-4 (middle) and hot core models 5-10 (bottom). The shaded region defines the upper and lower limit for the $\mathrm{HNCO} / \mathrm{CS}$ ratio as found by Martín et al. (2008).

processed. This is contrary to recent speculation about the origin of this species (Bisschop et al. 2007). The model also shows that in PDRs HNCO can not be directly produced in sufficient quantities to match observations. In hot cores in particular it seems that surface chemistry during an earlier cold collapse phase followed by gas-phase processing (in the post-collapse regime) is important in the production of HNCO.

The ratio of HNCO to CS evolves strongly as a function of time while its absolute value is a sensitive function of the sulphur abundance. Whilst observations have suggested this ratio can be used to trace different physical conditions, our findings show that metallicity, choice of chemical network and hot core age can all also contribute to this value. Indeed, considering that $\mathrm{HNCO}$ 
D. M. Tideswell et al.: The abundance of $\mathrm{HNCO}$ and its use as a diagnostic of environment

contains three heavy elements one may expect the abundance of $\mathrm{HNCO}$ to be highly sensitive to the metallicity of a region.

Acknowledgements. D.M.T. wishes to acknowledge the receipt of an STFC studentship. Astrophysics at the JBCA and QUB is supported by grants from the STFC. This work has also been supported by the European Communitys human potential programme under contract MCRTNCT-512302 (The Molecular Universe). The authors would like to thank Paul Woods for his excellent proof reading ability.

\section{References}

Allen, M., \& Robinson, G. W. 1977, ApJ, 212, 396

Anicich, V. G. 2004, JPL Publication 03-19

Bisschop, S. E., Jørgensen, J. K., van Dishoeck, E. F., et al. 2007, A\&A, 465, 913

Bronfman, L., Nyman, L. A., \& May, J. 1996, A\&AS, 115, 81

Brown, R. L. 1981, ApJ, 248, L119

Garrod, R. T., Weaver, S. L. W., \& Herbst, E. 2008, ApJ, 682, 283

Goldsmith, P. F., Snell, R. L., Deguchi, S., Krotkov, R., \& Linke, R. A. 1982, ApJ, 260, 147
Gredel, R., Lepp, S., Dalgarno, A., et al. 1989, ApJ, 347, 289

Habart, E., Abergel, A., Walmsley, C. M., Teyssier, D., \& Pety, J. 2005, A\&A, 437, 177

Hasegawa, T. I. \& Herbst, E. 1993, MNRAS, 261, 83

Hasegawa, T. I., Herbst, E., \& Leung, C. M. 1992, ApJS, 82, 167

Helmich, F. P., \& van Dishoeck, E. F. 1997, A\&AS, 124, 205

Iglesias, E. 1977, ApJ, 218, 697

Jackson, J. M., Armstrong, J. T., \& Barrett, A. H. 1984, ApJ, 280, 608

MacDonald, G. H., Gibb, A. G., Habing, R. J., et al. 1996, A\&AS, 119, 333

Martín, S., Requena-Torres, M. A., Martín-Pintado, J., et al. 2008, ApJ, 678, 245

Millar, T. J., \& Herbst, E. 1990, MNRAS, 242, 92

Nummelin, A., Bergman, P., Hjalmarson, A., et al. 2000, ApJS, 128, 213

Rawlings, J. M. C., Hartquist, T. W., Menten, K. M., et al. 1992, MNRAS, 255, 471

Roberge, W. G., Jones, D., Lepp, S., et al. 1991, ApJS, 77, 287

Ruffle, D. P., \& Herbst, E. 2000, MNRAS, 319, 837

Snyder, L. E., \& Buhl, D. 1972, ApJ, 177, 619

Spitzer, L. 1978, Physical processes in the interstellar medium, 2nd edn. (John Wiley and Sons)

Turner, B. E., Terzieva, R., \& Herbst, E. 1999, ApJ, 518, 699

Wilson, T. L., \& Rood, R. 1994, ARA\&A, 32, 191

Woodall, J., Agúndez, M., Markwick-Kemper, A. J., et al. 2007, A\&A, 466, 1197

Zinchenko, I., Henkel, C., \& Mao, R. Q. 2000, A\&A, 361, 1079 